\title{
Effect of Concomitant 3-Hydroxy-3-Methyl-Glutaryl- CoA Reductase Inhibitor Therapy on Creatine Phosphokinase Levels and Mortality Among Patients Receiving Daptomycin: Retrospective Cohort Study
}

Heather L. McConnell • Elizabeth T. Perris • Colleen Lowry •

Thomas Lodise $\cdot$ Nimish Patel

To view enhanced content go to www.infectiousdiseases-open.com

Received: July 9, 2014 / Published online: September 23, 2014

(c) The Author(s) 2014. This article is published with open access at Springerlink.com

\section{ABSTRACT}

Introduction: The prescribing information for daptomycin recommends discontinuing statin therapy during receipt of daptomycin. The literature supporting this recommendation is sparse. The objectives of this study were to examine the impact of 3-hydroxy-3-methylglutaryl-CoA (HMG-CoA) reductase inhibitors (statins) on creatine phosphokinase (CPK) elevations and mortality among patients receiving daptomycin therapy.

Methods: A retrospective cohort study was performed among daptomycin recipients in the Upstate New York Veterans' Healthcare Administration from September 15, 2003 to July 1, 2013. Inclusion criteria were: (1)

Electronic supplementary material The online version of this article (doi:10.1007/s40121-014-0041-y) contains supplementary material, which is available to authorized users.

H. L. McConnell · E. T. Perris · C. Lowry

Samuel S. Stratton VA Medical Center, Albany, NY, USA

T. Lodise $\cdot$ N. Patel $(\bowtie)$

Pharmacy Practice Department, Albany College of Pharmacy and Health Sciences, 106 New Scotland Avenue, Albany, NY 12208, USA

e-mail: nimish.patel@acphs.edu daptomycin for $\geq 48 \mathrm{~h}$, (2) availability of baseline CPK value and (3) >1 CPK level measurement taken while on therapy. The following were extracted from medical records: demographics, comorbidities, laboratory data, medication history (daptomycin, statins and concomitant drugs known to increase CPK), Acute Physiology and Chronic Health Evaluation (APACHE)-II score and vital status at 30 days. The exposure of interest was use of statins. The primary outcome was CPK elevation defined as a CPK value $\geq 3$ times the upper limit of normal (ULN) if baseline CPK was normal, and $\geq 5$ times ULN if baseline CPK was elevated. The secondary outcome was death within 30 days of commencing daptomycin.

Results: A total of 233 patients were included in this analysis. Among these patients, 53 received concomitant statin therapy. Most baseline clinical characteristics were similar between statin recipients and non-recipients. Five $(2.1 \%)$ patients experienced a CPK elevation; $3 / 53(5.7 \%)$ were statin recipients and $2 / 180(1.1 \%)$ received daptomycin alone $(p=0.08)$. All patients with CPK elevations had normal baseline CPK values. No effect modification was observed by use of other 
concomitant medications known to increase CPK values. Death was observed more frequently among statin non-recipients (17.2\%) than recipients $(9.4 \%)$.

Conclusions: Among patients receiving daptomycin, no significant difference was observed in frequency of CPK elevation between statin recipients and non-recipients.

Keywords: Daptomycin; Mortality; Myopathy; Outcomes; Statins; Toxicity

\section{INTRODUCTION}

Daptomycin is an antibiotic used to treat complicated infections caused by aerobic Gram-positive organisms, including methicillin-resistant Staphylococcus aureus (MRSA) and vancomycin-resistant Enterococcus (VRE) [1]. The use of this agent has somewhat been limited by its toxicity profile, specifically the potential to cause elevations in creatine phosphokinase (CPK) [1]. This is a meaningful outcome as CPK elevation may be indicative of skeletal muscle toxicity and, in extreme circumstances, life-threatening conditions such as rhabdomyolysis [2].

The potential to cause elevations in CPK may be enhanced when daptomycin exposure is altered (e.g., higher doses or diminished creatinine clearance) [3] or when used in combination with other agents that also increase CPK [1]. Among medications known to elevate CPK, HMG-CoA reductase inhibitors (statins) are commonly used [4]. The prescribing information for daptomycin specifically recommends discontinuing statin therapy during receipt of daptomycin [1]. However, the literature supporting this recommendation is sparse, and there has not been a thorough assessment to determine if there is an increased frequency of CPK elevations between statin recipients and non-recipients receiving daptomycin.

The primary objective of this study was to compare the incidence of CPK elevation between recipients and non-recipients of statins receiving daptomycin. The secondary objective was to assess the relationship between concomitant statin therapy and mortality.

\section{METHODS}

\section{Study Design and Population}

A retrospective cohort study was performed among patients in the Upstate New York Veterans' Affairs Healthcare Network or Veterans' Integrated Service Network (VISN)-2 from September 15, 2003 to July 1, 2013. Inclusion criteria were: (1) receipt of daptomycin for $\geq 48 \mathrm{~h}$, (2) availability of baseline CPK value and (3) >1 CPK level measurement taken while on therapy. Information was only gathered for patients receiving daptomycin for their first treatment course during the study period. This retrospective study was approved by the Samuel S. Stratton Veterans' Affairs Research and Development Committee and the Institutional Review Board.

The retrospective analysis in this article does not involve any new studies of human subjects performed by any of the authors.

\section{Data Collection}

Data were extracted by trained reviewers and included demographics, comorbidities, laboratory data including CPK level and creatinine clearance (CrCL) [5], previous 
hospitalizations within 90 days, inpatient location [intensive care unit (ICU) versus nonICU], concomitant medications associated with CPK elevations, length of hospital stay, CPK levels and mortality. With regard to daptomycin use, information collected included the dose, frequency (standard frequency of daptomycin dosing is every $24 \mathrm{~h}$ or every $48 \mathrm{~h}$ ) and duration of daptomycin therapy.

Demographic characteristics included age, sex, weight and body mass index. Data on comorbidities experienced by each patient were collected, and included organ transplant, musculoskeletal disease, hypertension, cancer, congestive heart failure, renal impairment, liver disease, chronic obstructive pulmonary disease, cerebrovascular accident, and alcoholism. Use of concomitant medications known to increase CPK was captured for each patient during the time of daptomycin administration. These medications included: corticosteroids, phenothiazine antipsychotics, antiretrovirals, colchicine, chloroquine, hydroxychloroquine and recreational drug use. The specific statin used during daptomycin therapy was documented.

Microbiologic culture data were collected prior to the start of daptomycin. The indication for treatment was categorized as skin and skin structure, osteomyelitis, endocarditis, bacteremia or empiric therapy. Severity of illness at the initiation of therapy was calculated using the Acute Physiology and Chronic Health Evaluation (APACHE)-II [6].

\section{Statin Exposure}

The exposure of interest in this study was the use of statin therapy while receiving daptomycin. The specific statins that were available during the study period were: simvastatin, atorvastatin, pravastatin, rosuvastatin, lovastatin, fluvastatin and pitavastatin.

\section{Outcomes}

The outcomes examined were: (1) CPK elevation and (2) death within 30 days of commencing daptomycin. The definition of CPK elevation was adapted from Bhavnani et al. [3] CPK levels were assessed from day 4 of daptomycin therapy to 3 days after daptomycin was discontinued. For patients with a normal CPK level at baseline, CPK elevation was defined as a level $\geq 3$ times the upper limit of normal (ULN). Based on the reference range at the Veterans' Affairs Medical Center, this corresponded to CPK values in excess of 696 units/L. For patients with an abnormal CPK level at baseline, CPK elevation was defined as a level $\geq 5$ times ULN $(1,160$ units/L). Both definitions were based on two sequential measurements. Stratified analyses were performed between patients with normal versus abnormal baseline CPK values and $\mathrm{CrCL} \geq$ versus $<30 \mathrm{~mL} / \mathrm{min}$.

\section{Statistical Analyses}

In the bivariate analyses, categorical variables were assessed using Chi square and Fisher's exact test. For continuous variables, Student's $T$ and Mann-Whitney $U$ tests were used. The log-rank test was used to compare frequency of CPK elevations, integrated over time, between statin recipients and non-recipients. Stratified analyses were performed to assess for effect modification. Stratum-specific measures of association were compared using the BreslowDay test. All analyses were performed using SPSS (IBM, v21.0, Chicago, USA) and SAS (SAS Institute, v9.1, Cary, USA). 


\section{RESULTS}

There were 350 patients who received daptomycin at a VISN-2 facility. There were 117 patients that were excluded because they did not receive daptomycin for $\geq 48 \mathrm{~h}$ or did not have a CPK level measurement taken while on therapy. Among the 233 patients that met inclusion criteria, 180 received daptomycin alone and 53 received daptomycin while receiving a statin.

The demographics and clinical characteristics of the study population are displayed in Table 1. Overall, the majority of these baseline characteristics were similar between the two groups. Mean \pm standard deviation (SD) age was higher $(p=0.02)$ for statin recipients $(69.7 \pm 9.8)$ than nonrecipients $(65.1 \pm 13.0)$. Mean \pm SD APACHE-II scores were also higher $(p=0.04)$ in statin recipients $(13.9 \pm 7.3)$ than non-recipients $(11.6 \pm 6.8)$. Patients with a higher degree of renal dysfunction at baseline were more likely to receive daptomycin while receiving a statin. With regard to daptomycin use, there were no statistically significant differences observed in dose and duration of daptomycin therapy between groups. The frequency of concomitant medication use associated with CPK elevations did not differ significantly $(p=0.15)$ between statin users $(20.8 \%)$ and non-users $(12.8 \%)$. No patients were receiving phenothiazines, chloroquine or hydroxychloroquine.

Hypertension, congestive heart failure, renal disease and diabetes were observed more frequently among statin recipients than nonrecipients. The indications for daptomycin use had comparable distributions between statin users and non-users and daptomycin was most commonly being used to treat bacteremia and skin and skin structure infections. The most frequently used statin was simvastatin (58.5\%), followed by atorvastatin (17.0\%), pravastatin (13.2\%) and rosuvastatin (11.3\%). No patients received lovastatin, fluvastatin or pitavastatin.

Study outcomes are described in Table 2. Elevations in CPK levels were observed in a total of five patients (2.1\%). Among these five patients, $3 / 53 \quad(5.7 \%)$ patients received daptomycin while receiving a statin, and 2/180 (1.1\%) received daptomycin alone $(p=0.08)$. No effect modification was observed by use of other concomitant medications known to increase CPK values. In the time-toevent analyses, there was no significant difference in CPK elevation over time between statin recipients and non-recipients $(p=0.14)$. All patients that had a CPK elevation had a normal baseline CPK value. Baseline creatinine clearance also did not affect the development of CPK elevations. Of patients with creatinine clearances $\geq 30 \mathrm{~mL} / \mathrm{min}, 2.2 \%$ experienced a CPK elevation versus $2.0 \%$ of patients with creatinine clearances $<30 \mathrm{~mL} / \mathrm{min}$. The comparison of CPK elevations between patients who received daptomycin while receiving a statin and daptomycin alone, within each of these creatinine clearance strata, did not differ significantly (Breslow-Day test $p$ value $=0.47)$. Although not statistically significant $(p=0.17)$, a higher frequency of death was observed for patients who did not receive concomitant statin therapy $(17.2 \%)$ versus those who were on statin therapy (9.4\%).

\section{DISCUSSION}

We performed a retrospective cohort study of Veterans' Affairs patients that received daptomycin therapy to assess the relationship between concomitant statin therapy and CPK elevation. We observed no significant difference 
Table 1 Bivariate analyses comparing clinical and demographic characteristics among Veterans' Affairs patients receiving daptomycin with or without a statin

\begin{tabular}{|c|c|c|c|}
\hline Covariate & $\begin{array}{l}\text { No statin } \\
(n=180)\end{array}$ & $\begin{array}{l}\text { Statin } \\
(n=53)\end{array}$ & $P$ value \\
\hline Mean age (SD), years & $65.1(13.0)$ & $69.7(9.8)$ & 0.02 \\
\hline Male sex, $n(\%)$ & $173(96.1)$ & $51(96.2)$ & 0.97 \\
\hline Mean weight (SD), $\mathrm{kg}$ & $93.2(27.2)$ & $93.9(27.1)$ & 0.88 \\
\hline BMI categories $\left(\mathrm{kg} / \mathrm{m}^{2}\right), n(\%)$ & & & 0.54 \\
\hline$<20$ & $14(7.8)$ & $2(3.8)$ & \\
\hline $20-25$ & $41(22.8)$ & $15(28.3)$ & \\
\hline $25-30$ & $45(25.0)$ & $17(32.1)$ & \\
\hline $30-35$ & $49(27.2)$ & $9(17.0)$ & \\
\hline $35-40$ & $13(7.2)$ & $4(7.5)$ & \\
\hline$\geq 40$ & $18(10.0)$ & $6(11.3)$ & \\
\hline Creatinine clearance $(\mathrm{mL} / \mathrm{min}), n(\%)$ & & & 0.04 \\
\hline$>90$ & $57(31.7)$ & $7(13.2)$ & \\
\hline $60-90$ & $44(24.4)$ & $13(24.5)$ & \\
\hline $30-60$ & $44(24.4)$ & $17(32.1)$ & \\
\hline$<30$ & $35(19.4)$ & $16(30.2)$ & \\
\hline Patients with any comorbidities associated with CPK elevations, $n$ (\%) & $172(95.6)$ & $52(98.1)$ & 0.40 \\
\hline Organ transplant, $n(\%)$ & $2(1.1)$ & $0(0)$ & 1.00 \\
\hline Musculoskeletal disease, $n$ (\%) & $13(7.2)$ & $3(5.7)$ & 1.00 \\
\hline Hypertension, $n(\%)$ & $135(75.0)$ & $52(98.1)$ & $<0.001$ \\
\hline Cancer, $n(\%)$ & $41(22.8)$ & $11(20.8)$ & 0.76 \\
\hline $\mathrm{CHF}, n(\%)$ & $25(13.9)$ & $18(34.0)$ & 0.001 \\
\hline Renal disease, $n(\%)$ & $59(32.8)$ & $27(50.9)$ & 0.02 \\
\hline Liver disease, $n(\%)$ & $26(14.4)$ & $6(11.3)$ & 0.51 \\
\hline COPD, $n(\%)$ & $38(21.1)$ & $12(22.6)$ & 0.81 \\
\hline Diabetes, $n(\%)$ & $84(46.7)$ & $37(69.8)$ & 0.003 \\
\hline CVA, $n(\%)$ & $19(10.6)$ & $10(18.9)$ & 0.11 \\
\hline Alcoholism, $n$ (\%) & $24(13.3)$ & $6(11.3)$ & 0.70 \\
\hline Prior hospitalization, $n(\%)$ & $106(58.9)$ & $34(64.2)$ & 0.49 \\
\hline Dialysis, $n(\%)$ & $13(7.2)$ & $4(7.5)$ & 1.00 \\
\hline Median (IQR) dose of daptomycin (mg/kg) & $6(5.7-6.3)$ & $5.9(4.9-6.2)$ & 0.15 \\
\hline
\end{tabular}


Table 1 continued

\begin{tabular}{|c|c|c|c|}
\hline Covariate & $\begin{array}{l}\text { No statin } \\
(n=180)\end{array}$ & $\begin{array}{l}\text { Statin } \\
(n=53)\end{array}$ & $P$ value \\
\hline $\begin{array}{l}\text { Patients receiving alternative daptomycin dosing frequency } \\
\text { (once every } 48 \mathrm{~h} \text { ), } n(\%)\end{array}$ & $47(26.1)$ & $16(30.2)$ & 0.56 \\
\hline Patients requiring a dose adjustment, $n(\%)$ & $50(27.8)$ & $16(30.2)$ & 0.73 \\
\hline Indication for daptomycin therapy, $n$ (\%) & & & 0.25 \\
\hline Skin/soft tissue & $53(29.4)$ & $17(32.1)$ & \\
\hline Osteomyelitis & $20(11.1)$ & $9(17.0)$ & \\
\hline Endocarditis & $17(9.4)$ & $8(15.1)$ & \\
\hline Empiric therapy & $14(7.8)$ & $1(1.9)$ & \\
\hline Bacteremia & $76(42.2)$ & $18(34.0)$ & \\
\hline Patients with MRSA, $n$ (\%) & $53(29.4)$ & $22(41.5)$ & 0.10 \\
\hline Patients with MSSA, $n$ (\%) & $20(11.1)$ & $6(11.3)$ & 0.96 \\
\hline Patients with VRE, $n(\%)$ & $64(35.6)$ & $11(20.8)$ & 0.04 \\
\hline Median (IQR) time to first daptomycin dose after culture, days & $4(2-9)$ & $4(2-8)$ & 0.90 \\
\hline Patients in the ICU at initiation of daptomycin, $n(\%)$ & $54(30.0)$ & $18(34.0)$ & 0.58 \\
\hline Mean (SD) APACHE-II score & $11.6(6.8)$ & $13.9(7.3)$ & 0.04 \\
\hline Median (IQR) baseline CPK value & $40(23-93)$ & $49(30-101)$ & 0.94 \\
\hline Patient use of other drugs that increase CPK, $n(\%)$ & $23(12.8)$ & $11(20.8)$ & 0.15 \\
\hline Antipsychotics, $n$ (\%) & $16(8.9)$ & $6(11.3)$ & 0.61 \\
\hline Colchicine, $n(\%)$ & $0(0)$ & $1(1.9)$ & 0.23 \\
\hline Corticosteroids, $n$ (\%) & $7(3.9)$ & $5(9.4)$ & 0.11 \\
\hline Antiretrovirals, $n(\%)$ & $1(0.6)$ & $0(0)$ & 1.00 \\
\hline Recreational drugs, $n(\%)$ & $37(20.6)$ & $12(22.6)$ & 0.71 \\
\hline
\end{tabular}

APACHE-II Acute Physiology and Chronic Health Evaluation II, BMI body mass index, CPK creatine phosphokinase, $C H F$ congestive heart failure, COPD chronic obstructive pulmonary disease, $C V A$ cerebrovascular accident, $I C U$ intensive care unit, IQR interquartile range, MRSA methicillin-resistant Staphylococcus aureus, MSSA methicillin-sensitive Staphylococcus aureus, SD standard deviation, VRE vancomycin-resistant Enterococcus

in the frequency of CPK elevation between statin recipients and non-recipients. Among patients with a creatinine clearance $>30 \mathrm{~mL} /$ min (same type of patients studied in registration trial [7] and Bhavnani et al. [3]), there was a lower incidence (2.2\%) of CPK elevation observed than previously reported in the literature [8-10], irrespective of whether daptomycin was given with or without a statin. Bhavnani et al. examined the probability of CPK elevations in patients who received daptomycin without a statin and determined the predicted probability of a CPK elevation to be between $3.73 \%$ and $19.5 \%$ (at doses ranging from 4 to 
Table 2 Clinical outcomes of statin users and non-users among Veterans' Affairs patients receiving daptomycin

\begin{tabular}{llll}
\hline Covariate & $\begin{array}{l}\text { No statin } \\
(\boldsymbol{n}=\mathbf{1 8 0})\end{array}$ & $\begin{array}{l}\text { Statin } \\
(\boldsymbol{n}=\mathbf{5 3})\end{array}$ & $\begin{array}{l}\boldsymbol{P} \\
\text { value }\end{array}$ \\
\hline CPK elevation, $n(\%)$ & $2(1.1)$ & $3(5.7)$ & 0.08 \\
Normal baseline CPK $(n=219), n(\%)$ & {$[n=170] 2(1.2)$} & {$[n=49] 3(6.1)$} & 0.08 \\
Abnormal baseline CPK $(n=14), n(\%)$ & {$[n=10] 0$} & {$[n=4] 0$} & N/A \\
$\begin{array}{l}\text { Median (IQR) day of daptomycin therapy where CPK elevation } \\
\text { occurred }\end{array}$ & $20(12-28)$ & $28(16-29)$ & 0.4 \\
$\begin{array}{l}\text { Median (IQR) peak CPK value on daptomycin therapy } \\
\text { Median (IQR) length of stay at medical center, days }\end{array}$ & $59(34-146)$ & $80(38-168)$ & 0.32 \\
Death, $n(\%)$ & $23(10-59)$ & $28(14-50)$ & 0.44 \\
\hline
\end{tabular}

Normal CPK level at baseline was defined as CPK elevation $\geq 3$ times the upper limit of normal (ULN) and corresponded to CPK values in excess of 696 units/L. Abnormal CPK level at baseline was defined as a level $\geq 5$ times ULN (1,160 units/ L)

$C P K$ creatine phosphokinase, $I Q R$ interquartile range

$12 \mathrm{mg} / \mathrm{kg} /$ day) [3]. It is interesting to note that the median (IQR) daptomycin dose in our study was $6.0(5.5-6.3) \mathrm{mg} / \mathrm{kg}$ and is similar to the actual dose $(6 \mathrm{mg} / \mathrm{kg})$ administered to patients in the bacteremia/endocarditis registration trial [7] and the simulations performed by Bhavnani et al. [3]. The lower frequency of CPK elevations may denote competing risks or an etiology that involves varying contributions of exposure, severity of illness and other clinical factors.

The results of our study are generally consistent with recently published data regarding lack of a statistically significant relationship between CPK elevations and concomitant use of daptomycin and statin therapy [2, 8-10]. Parra-Ruiz et al. reported an $8 \%$ occurrence of CPK elevation with the combination of high-dose ( $>6 \mathrm{mg} / \mathrm{kg} /$ day) daptomycin and statin therapy, and a $10 \%$ occurrence of CPK elevation in patients receiving daptomycin only [6]. While the point estimates are higher than those in our analysis, the comparison resulting in no difference between the two treatment groups is largely consistent.

Our study suggests that the incidence of CPK elevation may be less frequent than previously reported. This is an interesting finding given that the population studied included patients that received care at a VISN-2 facility. The Veterans' Affairs population is primarily made up of patients with a larger distribution of comorbidities than the general population [11]. Given their underlying health status, this is a population that one would expect to have a higher frequency of CPK elevations [12]. These findings have important implications for utilization of daptomycin since it may not be as toxic as previously perceived, regardless of whether used concomitantly with statin therapy. Future studies should assess the risk of CPK elevation in non-VA Medical Center settings where the volume of comorbidities is less pronounced.

Although not found to be statistically significant, daptomycin patients receiving a 
concomitant statin had a lower frequency of death versus statin non-recipients. These findings among daptomycin recipients are consistent with other retrospective studies that have shown beneficial effects with statin use in bacteremia, including reduction in all-cause mortality and bacteremia-related death [13, 14]. Given the lack of relationship between statin use and CPK elevation and the effect on mortality, the recommendations surrounding the concomitant statin use may warrant further investigation for daptomycin recipients.

Some considerations should be taken into account when interpreting these findings. First, only five CPK elevation events were observed and power may have been an issue indicating that a larger study needs to be performed. However, we evaluated all patients that received daptomycin since FDA approval in VISN-2. Over 500 patients would have been required to demonstrate a statistically significant difference in the small effect size (5.7\% versus $1.1 \%$ ) observed in our study and this was not feasible given daptomycin usage in VISN-2. Due to the very low number of events, we were unable to perform multivariate analysis to assess if statin therapy is independently associated with CPK elevation. Second, we were unable to discriminate the CPK risk associated with individual statins because the majority of patients were on the VA formulary agent, simvastatin, during the study period. Third, some patients who did not receive statin therapy while receiving daptomycin had previous exposure to statins. It is unclear whether previous exposure would affect the risk of developing a CPK elevation. Future studies should discern whether this is an important predictor. Fourth, this study was performed among patients receiving care at VISN-2 facilities. External validity may be an issue in populations with a different distribution of comorbidities and gender. Finally, none of the CPK events occurred in patients on hemodialysis. This is an important point to consider because these patients are likely to have altered drug exposure and potentially have a higher probability of achieving troughs in excess of $24.3 \mu \mathrm{g} / \mathrm{mL}$, the threshold associated with an elevated risk of CPK elevations [3]. Given that this trough target was derived from patients enrolled in registration trials of daptomycin, future studies should evaluate if this trough threshold is applicable to the general population of patients using daptomycin and if the threshold differs by use of concomitant statin therapy, hemodialysis and other clinical factors that may modify daptomycin exposure.

In summary, the incidence of CPK elevation did not significantly differ with or without concomitant statin therapy among patients receiving daptomycin. Previous recommendations to avoid this combination should be revisited given these findings. Future studies should examine this relationship in larger populations with more heterogeneous statin usage and attempt to quantify the risk of CPK elevation with individual statins.

\section{ACKNOWLEDGMENTS}

No funding or sponsorship was received for this study or publication of this article. All named authors meet the ICMJE criteria for authorship for this manuscript, take responsibility for the integrity of the work as a whole, and have given final approval for the version to be published. This material is based upon work partially supported by the Office of Research and Development, Department of Veterans' Affairs. 
Conflict of interest. Heather McConnell, Elizabeth Perris, Colleen Lowry and Nimish Patel declare that they have no conflicts of interest. Thomas Lodise is a consultant and grant recipient from Cubist Pharmaceuticals.

Compliance with ethics guidelines. This article does not contain any new studies with human or animal subjects performed by any of the authors.

Open Access. This article is distributed under the terms of the Creative Commons Attribution Noncommercial License which permits any noncommercial use, distribution, and reproduction in any medium, provided the original author(s) and the source are credited.

\section{REFERENCES}

1. Cubicin-Full Prescribing Information. Lexington, MA: Cubist Pharmaceuticals Inc.; 2013.

2. Golightly LK, Barber GR, Barron MA, Page RL 2nd. Statins and daptomycin: safety assessment of concurrent use and evaluation of drug interaction liability. Drug Metabol Drug Interact. 2013;28(1): 49-58.

3. Bhavnani SM, Rubino CM, Ambrose PG, Drusano GL. Daptomycin exposure and the probability of elevations in the creatine phosphokinase level: data from a randomized trial of patients with bacteremia and endocarditis. Clin Infect Dis. 2010;50(12): 1568-74.

4. Pencina MJ, Navar-Boggan AM, D'Agostino RB Sr, Williams K, Neely B, Sniderman AD, et al. Application of new cholesterol guidelines to a population-based sample. N Engl J Med. 2014; 370(15):1422-31.
5. Cockcroft DW, Gault MH. Prediction of creatinine clearance from serum creatinine. Nephron. 1976; 16(1):31-41.

6. Knaus WA, Draper EA, Wagner DP, Zimmerman JE. APACHE II: a severity of disease classification system. Crit Care Med. 1985;13(10):818-29.

7. Fowler VG Jr, Boucher HW, Corey GR, Abrutyn E, Karchmer AW, Rupp ME, et al. Daptomycin versus standard therapy for bacteremia and endocarditis caused by Staphylococcus aureus. N Engl J Med. 2006;355(7):653-65.

8. Berg ML, Estes LL, Dierkhising RA, Curran B, Enzler MJ. Evaluation of impact of statin use on development of CPK elevation during daptomycin therapy. Ann Pharmacother. 2014;48(3):320-7.

9. Bland CM, Bookstaver PB, Lu ZK, Dunn BL, Rumley KF. Musculoskeletal Safety Outcomes of Patients Receiving Daptomycin with HMG-CoA Reductase Inhibitors. Antimicrob Agents Chemother. $2014 \mathrm{Jul}$ 14.

10. Parra-Ruiz J, Duenas-Gutierrez C, Tomas-Jimenez C, Linares-Palomino JP, Garrido-Gomez J, HernandezQuero J. Safety analysis of high dose $(>6 \mathrm{mg} / \mathrm{kg} /$ day) daptomycin in patients with concomitant statin therapy. Eur J Clin Microbiol Infect Dis. 2012;31(8):1771-4.

11. Agha Z, Lofgren RP, VanRuiswyk JV, Layde PM. Are patients at Veterans Affairs medical centers sicker? A comparative analysis of health status and medical resource use. Arch Intern Med. 2000;160(21): 3252-7.

12. Rosenson RS. Current overview of statin-induced myopathy. Am J Med. 2004;116(6):408-16.

13. Kruger P, Fitzsimmons K, Cook D, Jones M, Nimmo G. Statin therapy is associated with fewer deaths in patients with bacteraemia. Intensive Care Med. 2006;32(1):75-9.

14. Mekontso Dessap A, Ouanes I, Rana N, Borghi B, Bazin C, Katsahian S, et al. Effects of discontinuing or continuing ongoing statin therapy in severe sepsis and septic shock: a retrospective cohort study. Crit Care. 2011;15(4):R171. 